\title{
Ultrasonic Investigation of Snow and Ice Parameters
}

\author{
T. GUDRA* AND L. NAJWER \\ Institute of Telecommunications, Teleinformatics and Acoustics, Wrocław University of Technology \\ Wybrzeże Wyspiańskiego 27, 50-370 Wrocław, Poland
}

In the paper some ultrasonic methods of measurement of sound velocity and attenuation in snow and ice media are presented. The measurement setup consists of ultrasonic airborne transducers working at the frequency of $f=40 \mathrm{kHz}$. A non-contact method of reflection coefficient on air-snow border was applied during measurement of different types of snow in the density range of $120-700 \mathrm{~kg} / \mathrm{m}^{3}$. Examples of obtained signals are also presented. It was proved that there is a linear dependence between reflection coefficient and snow density. The state of snow or ice layer is not precisely correlated with their acoustical parameters: ultrasound wave velocity and attenuation of ultrasonic wave during the measurement of the layer depth using the non-contact method. Reflection coefficient of snow can be used for estimation of snow density.

PACS: 42.68.Ge, 42.79.Ls, 43.35.Ae, 92.60.Wc

\section{Introduction}

Development of ultrasonic techniques for measuring various media broadens the knowledge about the parameters of the milieux and allows automation of such measurements. Previous attempts to determine the parameters of snow and ice can be divided into procedures utilizing acoustic, ultrasonic and optical waves. Due to the nature of those media, acoustic methods (including ultrasonic) seem most interesting because, e.g. acoustic/ ultrasonic waves can propagate in opaque environments.

\section{Physical properties of snow}

Solid and liquid precipitation is produced at altitudes at which atmosphere becomes saturated with water vapour, i.e. above lifted condensation level. The complex microphysical processes that occur in clouds produce droplets and snow crystals. Most of the crystal nuclei are created around freezing nuclei, which are particles from the ground (such as, e.g. soil particles lifted to high altitudes due to turbulent flow). Snowflake is formed as a result of sublimation and coagulation. The biggest snowflakes fall to the bottom of a cloud reaching a layer mostly filled with supercooled droplets. As an effect of sublimation snowflakes increase their size, leave the cloud and fall to the ground creating snow cover.

Snow cover constantly changes, influenced by internal and external factors. During snowfall the shape of snow cover is mostly affected by temperature. Below $-10^{\circ} \mathrm{C}$ snow forms a fluffy layer (powder), between $-10^{\circ} \mathrm{C}$ and $-3{ }^{\circ} \mathrm{C}$ snowflakes stick together and form packed powder and above $-3^{\circ} \mathrm{C}$ snowflakes clump together and stick to the ground forming wet snow. Wind, temperature, radiation and air humidity are the external factors, while pressure exerted by each individual snow layer and temperature inside snow cover can be classified as internal factors. Low air temperature causes contraction of snow

\footnotetext{
* corresponding author; e-mail: Tadeusz.Gudra@pwr.wroc.pl
}

masses, as a result of which gaps and cracks form inside snow cover. Above zero temperature over a longer period of time causes formation of packing snow as a result of water from the melted snow percolating into the lower layers. Depth hoar is formed when water vapour from the warmer levels of snow cover near the ground rises to the higher levels, where snow crystals are formed. Melting and freezing of the internal layers of snow, caused by heat penetrating the cover, results in formation of firn.

Specific gravity for individual snow types [1]:

$$
\begin{aligned}
& \text { - fresh snow (powder) - about } 1 \mathrm{kN} / \mathrm{m}^{3} \text {, } \\
& \text { — packed powder - about } 2 \mathrm{kN} / \mathrm{m}^{3} \text {, } \\
& \text { — packing snow - about } 4 \mathrm{kN} / \mathrm{m}^{3} \text {. }
\end{aligned}
$$

\section{Physical properties of ice}

Ice is formed in the process of water freezing, which occurs at $0^{\circ} \mathrm{C}$ temperature and $101325 \mathrm{~Pa}$ atmospheric pressure [2]. Maximum water density occurs at $4^{\circ} \mathrm{C}$. Further cooling results in decreasing density. During freezing, the density of water is lower by about $10 \%$, which is the reason why ice floats on water. Ice also forms outside water basins, e.g. in atmosphere (ice needles, snow pellets or hail) or on the ground (glaze). As in the case of snow cover, there are various types of ice, differentiated depending on the way it is formed. Snow ice forms when snow falls on water surface at a temperature of about $0^{\circ} \mathrm{C}$ — ice does not melt. Sudden temperature drop causes snow ice to change into white ice. Supercooled water and low air temperature are ideal conditions for the formation of frazil ice, small crystals of free-floating ice. It adheres to objects in the water or causes frazil jams and as a result causes serious operational problems in case of water intakes, hydro-electric power plants, water reservoirs and dam facilities. Growing frazil areas gradually turn into solid ice cover that has uneven and heterogeneous surface. Specific gravity of ice is $900 \mathrm{kN} / \mathrm{m}^{3}$. 


\section{Standard measurements of snow layer}

Development in the field of automation of measurement of snow layer parameters [3] allows us more detailed and frequent observations for meteorological, climatological and hydrological purposes as well as for the use of tourists and skiers. Snow cover measurements which are a part of standard meteorological observation are performed everyday from its appearance to the day it completely disappears. The following parameters are measured in specifically selected areas:

- thickness of the layer of snow that fell since the previous observation,

- total volume of all fall since the previous observation,

- total depth of all the snowfall,

- water equivalent of snow that fell since the previous observation,

— total water equivalent of all the snowfall.

One of the major parameters in avalanche forecasting is snow density which can be determined on the basis of the value of water equivalent for fresh snow. Unfortunately, previous methods of measuring this parameter only involve examining snow layer that was collected in a special tank of the measurement device. Such a measurement is only genuine when there is absolute certainty that the tank contains just the fresh snowfall (i.e. there were no winds that could move snow from the surrounding area, there was no melting or other types of fall during measurement). Developing a method for fast and unequivocal determination of the density of snow cover, that additionally would not require human involvement, would facilitate performing more frequent and precise measurements. The total depth of snow layer is the only parameter that has successfully been measured automatically using ultrasonic technology.

Between 1998 and 1999 basic acoustic properties of snow layer were examined [4]. Measurements were performed in acoustic frequency band. They involved such parameters as propagation velocity and attenuation of ultrasonic wave in snow. The measured velocity value began at $100 \mathrm{~m} / \mathrm{s}$ for frequency $f=50 \mathrm{~Hz}$ and tended towards sound velocity in air with increasing frequencies. Absorption coefficient in snow layer was very high and exceeded 0.9 for the whole frequency range of $50 \mathrm{~Hz}-5 \mathrm{kHz}$ that was used for the tests. Unfortunately, experiments were performed for just one snow density. It is, therefore, impossible to use the results presented in the study to determine any relations allowing associating physical properties of snow with its acoustic features. Additionally, the measurement method used in the work is questionable, especially in relation to sound wave propagation velocity measurements. Results obtained using this method suffer from significant measuring error that results from the way phase velocity was measured.

\section{Standard measurements of ice}

Ice is heterogeneous by nature; assessment of it is difficult and there are no established methods of measuring its parameters. The most common examination of ice is measurement of the thickness of its layer in a water basin. Until recently standard observation only involved thickness measurement and assessment of the character of ice cover.

In 1988 and 1990 researchers at the Applied Physics Laboratory of the University of Washington studied acoustic properties of arctic ice $[5,6]$. The experiments were aimed at relating acoustic properties of ice with its physical features. Research was done using ultrasonic waves in the frequency range of $37-150 \mathrm{kHz}$. The measurements included propagation velocity and attenuation of ultrasonic wave in various ice layers as well as reflection coefficient of wave inside ice layer.

\section{Measurement of snow parameters}

Among the various techniques of measuring propagation velocity of ultrasonic wave [7] reflection method (one sending and receiving probe used) and transmission method (a pair of probes used: a sending and a receiving one) are both suitable in case of assessment in snow and ice. Additionally, in both contact and non-contact method of measuring the time it takes a pulse to pass through the studied snow or ice layer can be used. Figure 1 shows a technique of measuring ultrasonic pulse delay using one sending and receiving probe and lists appropriate relations that facilitate determination of ultrasonic wave propagation velocity.

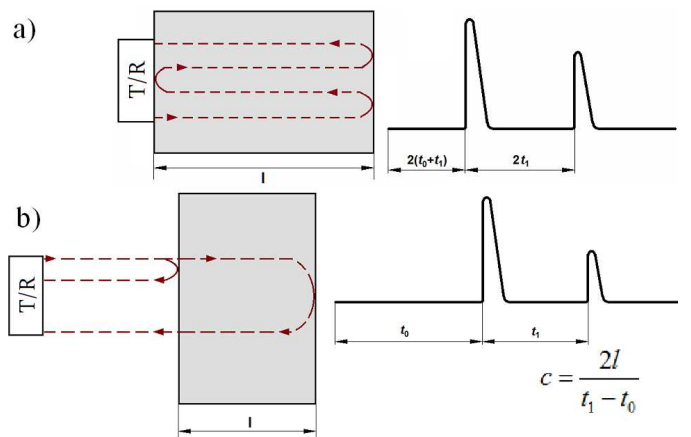

Fig. 1. The method of measuring ultrasonic pulse delay using one sending-receiving probe: (a) contact measurement, (b) non-contact measurement (based on [7]).

Figure 2 shows a technique of measuring ultrasonic pulse delay using a sending and a receiving probe.

Since it is necessary to maintain stable measurement conditions, the non-contact method was selected for snow assessment. An ultrasonic transducer intended to operate in air environment at the frequency of $f=40 \mathrm{kHz}$ was used during the tests. The level of sound pressure generated by the sending transducer was about $120 \mathrm{~dB}$. The sensitivity of a receiving transducer was $-59 \mathrm{~dB} / \mathrm{V} / \mu$ bar. 


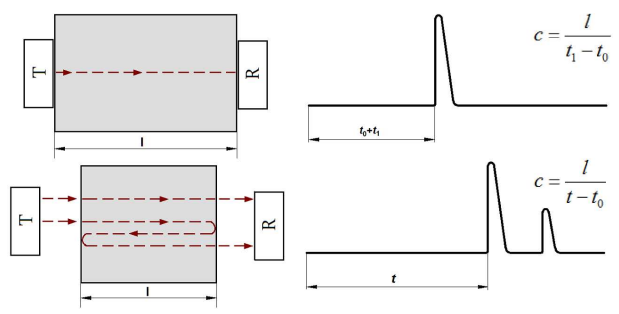

Fig. 2. The method of measuring ultrasonic pulse delay using two probes: a sending and a receiving one (based on [7]).

Figure 3 shows examples of oscillograms of ultrasonic pulses recorded after they have passed through $l=100 \mathrm{~mm}$ of air and through snow layers of the same thickness but various densities. Table I shows the determined values of ultrasonic wave propagation velocity for those types of snow.
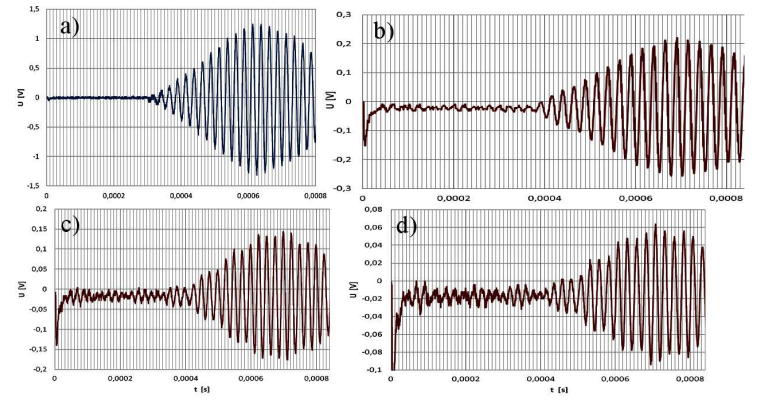

Fig. 3. Oscillograms of ultrasonic pulses recorded after they have passed through $l=100 \mathrm{~mm}$ thick layer: (a) in air environment, (b) in snow of density $\rho=120 \mathrm{~kg} / \mathrm{m}^{3}$, (c) in snow of density $\rho=170 \mathrm{~kg} / \mathrm{m}^{3}$, (d) in snow of density $\rho=222 \mathrm{~kg} / \mathrm{m}^{3}$.

TABLE I

Comparison of ultrasonic wave propagation velocity in snow that has various densities.

\begin{tabular}{c|c}
\hline \hline $\begin{array}{c}\text { Snow density } \\
{\left[\mathrm{kg} / \mathrm{m}^{3}\right]}\end{array}$ & $\begin{array}{c}\text { Wave velocity } \\
{[\mathrm{m} / \mathrm{s}]}\end{array}$ \\
\hline 120 & 264.4 \\
170 & 258.9 \\
222 & 253.7
\end{tabular}

In order to determine attenuation coefficient in snow the researchers measured the decrease in the level of signal on an $l=80 \mathrm{~mm}$ and $l=100 \mathrm{~mm}$ long path in air and in snow layers that had corresponding thickness values. All the measurements were performed in an arrangement identical to the one for assessment of propagation velocity of ultrasonic wave. Table II shows the determined values of attenuation coefficients for air and for various densities of snow measured during propagation of ultrasonic wave, the frequency of which was $f=40 \mathrm{kHz}$.

\section{TABLE II}

Attenuation coefficient for air and various snow densities.

\begin{tabular}{c|c}
\hline \hline Medium & $\begin{array}{c}\text { Attenuation } \\
{[\mathrm{dB} / \mathrm{cm}]}\end{array}$ \\
\hline air & 0.12 \\
snow $-\rho=120 \mathrm{~kg} / \mathrm{m}^{3}$ & 0.85 \\
snow $-\rho=170 \mathrm{~kg} / \mathrm{m}^{3}$ & 1.02 \\
snow $-\rho=222 \mathrm{~kg} / \mathrm{m}^{3}$ & 1.33
\end{tabular}

In order to determine the coefficient of reflection off the surface of snow which has various densities it was necessary to design a method that allows repeatable measurement of the signal reflected from the surface of various snow types. Figure 4 shows a picture of a setup for measuring and monitoring the path of signal reflected from the surface of snow.

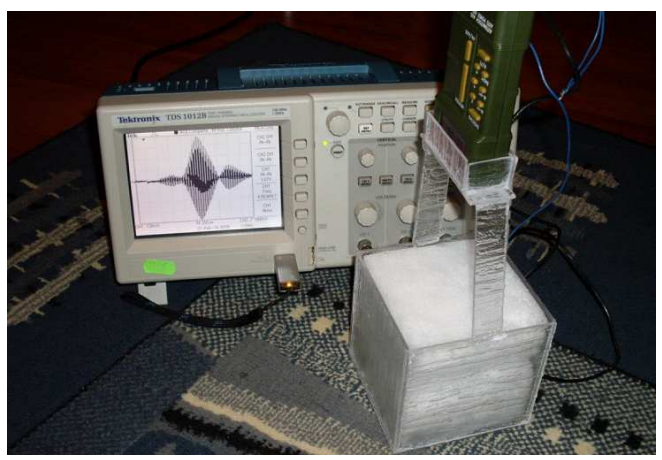

Fig. 4. Picture of a setup for measuring signal reflected from the surface of snow.

The $1 \mathrm{dm}^{3}$ cubic container visible in the picture was made of acrylic glass (a material with low thermal conductivity coefficient) and was used to collect sufficient amount of snow without changing its density. A sending and a receiving ultrasonic transducer are located at the height of $h=100 \mathrm{~mm}$, perpendicularly to the surface of snow. The reflected signal was monitored on the oscilloscope screen. The setup recorded peak to peak voltage amplitude $U_{\mathrm{pp}}$. In order to check repeatability of the results each snow type was tested several times. The research team measured the mass of each type of snow using an electronic weather. The reflection coefficient was determined by comparing signals reflected from the surface of snow with the signal reflected from the surface of water (reference signal - see Fig. 5a). Examination of each type of snow was performed in the field and the weather was the same as when the samples were collected. This solution had a clear advantage: no unwanted snow melting during assessment and ability to measure reference signal in identical weather conditions. 

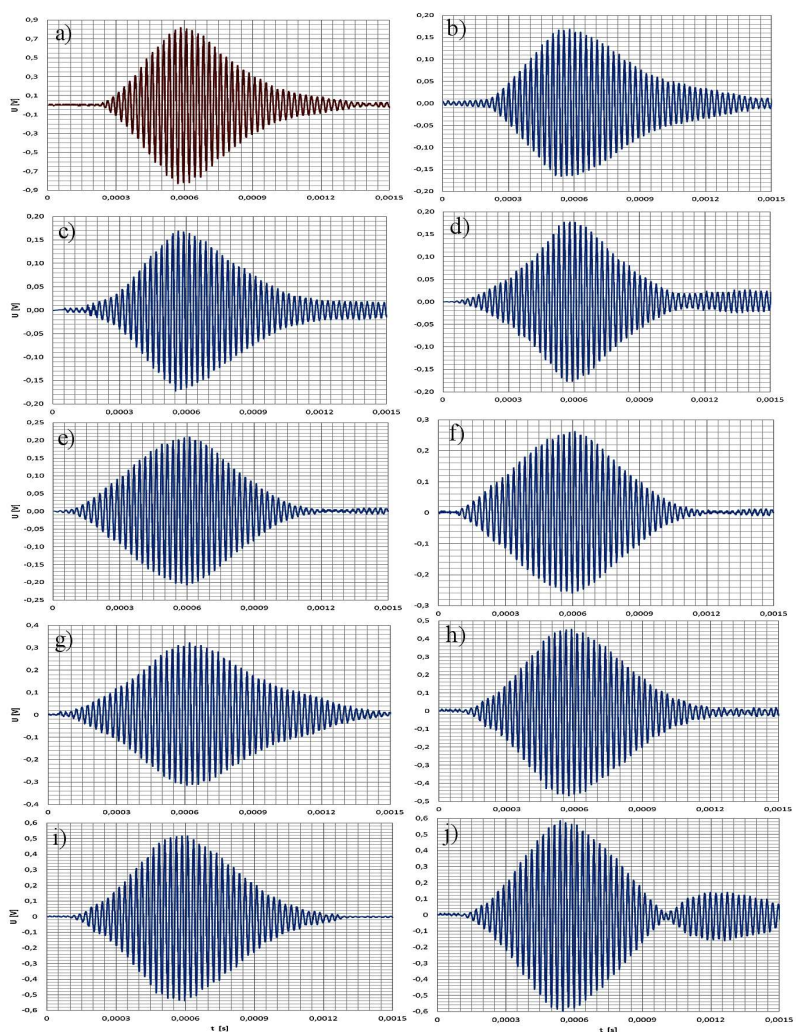

Fig. 5. Ultrasonic pulses $(f=40 \mathrm{kHz})$ reflected from: (a) surfaces of water $(R=100 \%)$, (b) surface of snow of density $\rho=120 \mathrm{~kg} / \mathrm{m}^{3}(R=20.3 \%)$, (c) $\rho=124 \mathrm{~kg} / \mathrm{m}^{3}$ $(R=20.6 \%),(\mathrm{d}) \rho=132 \mathrm{~kg} / \mathrm{m}^{3} \quad(R=22.19 \%),(\mathrm{e})$ $\rho=170 \mathrm{~kg} / \mathrm{m}^{3} \quad(R=20.77 \%),(\mathrm{f}) \rho=232 \mathrm{~kg} / \mathrm{m}^{3}$ $(R=31.88 \%),(\mathrm{g}) \rho=325 \mathrm{~kg} / \mathrm{m}^{3} \quad(R=37.24 \%)$, (h) $\rho=495 \mathrm{~kg} / \mathrm{m}^{3}(R=53.29 \%)$, (i) $\rho=591 \mathrm{~kg} / \mathrm{m}^{3}$ $\left(R=63.19 \%\right.$, (j) $\rho=696 \mathrm{~kg} / \mathrm{m}^{3}(R=70.24 \%)$.

Figure 6 shows the characteristics of wave reflection coefficient in snow density function. As expected, the obtained relation has a near linear character; approximation of the diagram to $1000 \mathrm{~kg} / \mathrm{m}^{3}$ density corresponds to the near maximum value of the reflection coefficient.

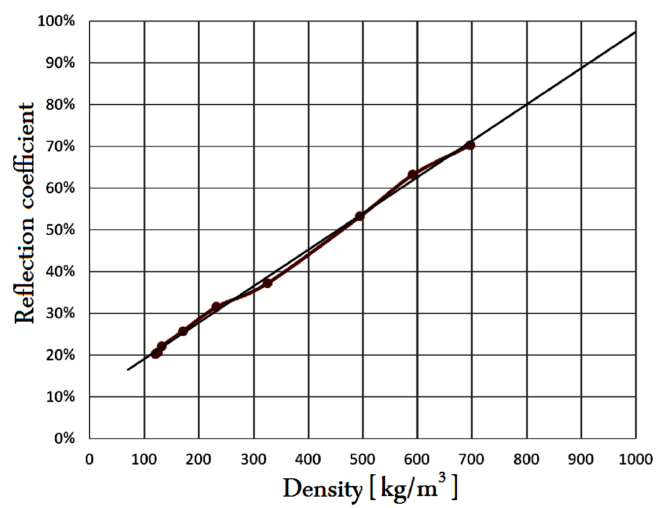

Fig. 6. Reflection coefficient vs. snow density.

\section{The method of measuring ice parameters}

Ultrasonic wave propagation velocity in ice was determined by means of measuring pulse delay with the use of two ultrasonic probes submerged in water and located at a distance from the ice sample as in the arrangement visible in Fig. 7.

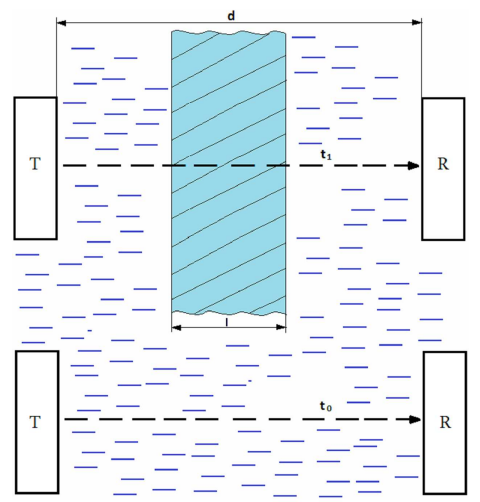

Fig. 7. Assessment of ultrasonic wave propagation velocity in ice using the method of measuring pulse delay (based on [7]).

In this type of measurement a sample is placed in a very thin insulation coating and submerged in water between the sending and receiving probe so that it does not touch the probes. After pulse passage time along water-studied sample-water path was measured, the ice sample was removed from water and ultrasonic pulse passage time between the sending and receiving probe was checked. Ultrasonic wave propagation velocity in ice was determined using the following dependence:

$$
c=\left(\frac{1}{c_{0}}-\frac{t_{0}-t}{l}\right)^{-1},
$$

where $c_{0}$ - ultrasonic wave propagation velocity based on time $t_{0}$ measured in water, $t-$ pulse passage time through ice sample submerged in water between probes, $l$ - thickness of the studied ice sample.

Water temperature during the test was $+5^{\circ} \mathrm{C}$. Ultrasonic wave propagation velocity in water with known temperature was determined using the following formula [8]:

$$
\begin{aligned}
c & =1.402385 \times 10^{3}+5.038813 T-5.799136 \times 10^{-2} T^{2} \\
& +3.287156 \times 10^{-4} T^{3}-1.398845 \times 10^{-6} T^{4} \\
& +2.78786 \times 10^{-9} T^{5} .
\end{aligned}
$$

The above formula is valid in temperature range of $0{ }^{\circ} \mathrm{C}-95^{\circ} \mathrm{C}$, for mean atmospheric pressure. For water temperature of $+5^{\circ} \mathrm{C}$ ultrasonic wave propagation velocity in water is about $1426 \mathrm{~m} / \mathrm{s}$. Ultrasonic probes operating at the frequency of $f=3 \mathrm{MHz}$ were used during the measurement. During the test the distance between the probes was $l=100 \mathrm{~mm}$. The measured pulse passage time between the probes with no ice sample between them was $t_{0}=70 \mu \mathrm{s}$; with the sample between the probes 
it decreased to $t=58 \mu \mathrm{s}$. Ultrasonic wave propagation velocity calculated using formula (1) was $c=3894 \mathrm{~m} / \mathrm{s}$ and is near the mean velocity values provided in literature $[5,6]$.

Attenuation coefficient in ice layer was determined by means of the same arrangement as the one shown in Fig. 7. The study was performed on two samples with parallel walls and thickness of $l=10 \mathrm{~mm}$ and $l=20 \mathrm{~mm}$. Based on the measurement of the level of signal received by the receiving probe in a setup: with no ice sample and with an ice sample with $l=10 \mathrm{~mm}$ and $l=20 \mathrm{~mm}$ thickness the researchers calculated attenuation coefficient in water, which for the measurement frequency of $f=3 \mathrm{MHz}$ was $0.7 \mathrm{~dB} / \mathrm{mm}$.

The measurement of coefficient of reflection from the surface of ice was performed in a similar fashion as in case of snow (i.e. using an ultrasonic transducer operating in air at the frequency of $f=40 \mathrm{kHz}$ ). The study of this parameter was performed for ice produced by freezing chlorinated water, mineral water and water collected from Oder river. All the measurements showed that the amplitude of signal after it is reflected off the surfaces of the studied ice "types" is similar and does not differ from the signals obtained after reflection of the surface of water.

\section{Conclusions}

The study results presented in the work prove that some of the known ultrasonic methods of measuring the properties of solid bodies and liquids can be successfully used to determine some properties of snow and ice. Because of the physical properties of snow, its parameters change with time and as a result of changing weather. The most valuable information about snow cover are its density and depth. Ultrasonic technology allows e.g. remote monitoring of snow cover depth or even automation of the process of measuring its parameters - no human involvement required during everyday monitoring on meteorological stations. This would facilitate increased number of measurement points and significant reduction of time required to perform such tests. It seems that measuring the coefficient of reflection of ultrasonic wave from the surface of snow is essential in this case. The possibility of performing fast and continuous assessment of this parameter using an aero-location device allows associating the coefficient of reflection from the surface of snow with snow density. The performed measurements show that it is a linear dependence, which significantly simplifies interpretation of the obtained results and can be useful, e.g. in determining roof loads of various structures - data essential in order to assess the risk of often tragic construction disasters.

Due to its physical characteristics ice is a medium the properties of which do not change to the same extent as in case of snow. Research results presented in the work show that tests of ultrasonic wave propagation velocity, attenuation or coefficient of reflection from the surface of ice does not provide useful data related to the type or condition of an ice layer. The performed tests were intended to check the available methods of measuring ice parameters and compare the results with those provided in literature (especially in case of ultrasonic wave propagation velocity).

\section{References}

[1] R. Skapski, About Snow, www.imgw.pl (in Polish).

[2] R. Skapski, Ice Phenomena, www.imgw.pl (in Polish).

[3] N.J. Doesken, R.J. Leffler, "Snow foolin", Weatherwise, (Jan/Feb 2000).

[4] T. Iwase, T. Sakuma, K. Yoshihisa, in: Proc. 17th Int. Cong. Acoust., Rome, Vol. 1, Ed. A. Bettucci, ICA Rome 2001, p. 274

[5] G.R. Garrison, R.E. Francois, T. Wen, R.P. Stein, W.J. Felton, "Sound Speed, Reflectivity and Absorption Measurements in Arctic Ice in 1988", Technical Report of Washington Univ. Seattle, Applied Physics Lab, Mar. 1991.

[6] G.R. Garrison, K.L. Williams, P.D. Mourad, R.E. Francois, T. Wen, W.J. Felton, "Sound Speed, Reflectivity, Absorption, and Thermal Diffusivity Measurements in Arctic Ice in 1990", Technical Report of Washington Univ. Seattle, Applied Physics Lab, Nov. 1993.

[7] J. Wehr, Measurements of Velocity and Attenuation of Ultrasonic Waves, PWN, Warsaw 1972 (in Polish).

[8] Underwater Acoustics, Technical Guides - Speed of Sound in Pure Water, National Physical Laboratory, Crown 2000 